Artículo Original. Enero-Abril 2017; 7(1):44-47. Recibido: 15/01/2017 Aceptado: 07/03/2017.

http://dx.doi.org/10.21929/abavet2017.71.4

\title{
Evaluación de parámetros productivos y rendimiento de la canal de conejos que consumieron infusión de epazote (Chenopodium ambrosioides).
}

Evaluation of productive parameters and carcass yield of rabbits, that consumed epazote (Chenopodium ambrosioides) infusion.

\author{
García-Vázquez Luisa ibt.garval@gmail.com, Ayala-Martínez \\ Maricela ayalam@uaeh.edu.mx, Zepeda-Bastida \\ Armando azepeda@uaeh.edu.mx,Ojeda-Ramírez \\ Deyaniradojeda@uaeh.edu.mx, Soto-Simental Sergio*sotos@uaeh.edu.mx
}

Instituto de Ciencias Agropecuarias, Universidad Autónoma del Estado de Hidalgo. México. *Autor responsable y de correspondencia: Soto-Simental Sergio, Instituto de Ciencias Agropecuarias, Universidad Autónoma del Estado de Hidalgo. Ave. Universidad s/n km 1. Tulancingo, Hidalgo. México.

\section{RESUMEN}

La cunicultura es una actividad que está creciendo a nivel mundial en los últimos tiempos, debido a su fácil manejo, la rapidez con la que se reproducen y la posibilidad de generar animales para su venta 0 autoconsumo. Uno de los principales problemas a los que se enfrenta esta actividad, es que no hay opciones farmacológicas para el tratamiento de enfermedades en dichos animales, por tal motivo, se han buscado estrategias alternativas para proteger y/o prevenir contra estas enfermedades, una de las opciones es el uso de plantas medicinales. Aquí evaluamos el efecto de la utilización de infusión de epazote en la engorda de conejos, sobre la producción animal y rendimiento de la canal. Los resultados encontrados mostraron que el uso de $10 \mathrm{~g}$ de epazote administrados como infusión en la engorda de conejos, aumentó los parámetros productivos y permitió que no hubiera mortalidad durante las 4 semanas de tratamiento, por lo que sería importante evaluar su efecto sobre la calidad de la carne y los productos cárnicos derivados de la misma.

Palabras clave: producción de conejo, rendimiento de la canal, Chenopodium ambrosioides

\begin{abstract}
Rabbit breeding is an activity that is growing worldwide in recent times, due to its easy handling, the speed with which they reproduce and the possibility of generating animals for sale or self-consumption. One of the main problems facing this activity is that there are no pharmacological options for the treatment of diseases in these animals, for that reason, alternative strategies have been sought to protect and/or prevent against these diseases, one of the options is the use of medicinal plants. Here we evaluate the effect of epazote infusion on rabbit fattening, animal production, and carcass yield. The results showed that the use of $10 \mathrm{~g}$ of epazote administered as an infusion in rabbit fattening increased the productive parameters and allowed no mortality during the 4 weeks of treatment, so it would be important to evaluate its effect on the quality of meat and meat products derived from it.
\end{abstract}

Keywords: Rabbit production, carcass yield, Chenopodium ambrosioides. 


\section{INTRODUCCIÓN}

La cunicultura es una actividad favorable para pequeños y medianos productores en todo el mundo, debido a su fácil manejo, la rapidez con la que se reproducen y la posibilidad de generar animales para autoconsumo o venta al público, para la obtención de ingresos económicos durante todo el año; aunado a lo anterior, la FAO (2015) menciona que su carne es muy nutritiva, tiene alto contenido en proteínas, poca grasa, es hipoalergénica y de alta digestibilidad (Para, 2015). Pese a estas ventajas, en México el progreso de la cunicultura se ha visto limitado, sobre todo por falta de apoyo gubernamental, carencia de políticas sanitarias que eviten las epizootias, poco interés en las instituciones de enseñanza e investigación, ausencia de animales genéticamente mejorados, poca difusión de los beneficios del consumo de esta carne y la poca organización que existe entre los productores (Olivares et al., 2015); por tal motivo, es necesario desarrollar investigaciones que fortalezcan el manejo productivo y sanitario de esta especie, su alimentación; así como implementar estrategias para mejorar el rendimiento económico. El objetivo de este estudio fue evaluar el efecto de la utilización de infusión de epazote en la engorda de conejos, sobre la producción animal y rendimiento de la canal.

\section{MATERIAL Y MÉTODOS}

Se utilizaron 18 conejos de $35 \mathrm{~d}$ de edad, con un peso vivo de $0.670 \mathrm{~kg}$ promedio, asignados completamente al azar en tres tratamientos: T1 $0 \mathrm{~g} \mathrm{~L}^{-1}$, T2 $5 \mathrm{~g} \mathrm{~L}^{-1}$ y T3 $10 \mathrm{~g} \mathrm{~L}^{-}$

${ }^{1}$ de epazote en infusión; los animales consumieron además alimento comercial a libre acceso. El consumo de alimento y agua fueron medidos diariamente, los animales fueron pesados una vez por semana. Después de $28 \mathrm{~d}$ de engorda los animales fueron transportados al Taller de Cárnicos del Instituto de Ciencias Agropecuarias, donde fueron sacrificados de acuerdo a la NOM-033-SAG/ZOO 2015, la canal fue diseccionada de acuerdo a las recomendaciones de Blasco y Ouhayoun (1996), para lo cual se pesó la canal caliente.

Para el análisis estadístico se empleó un diseño completamente al azar, cuando se encontraron diferencias se utilizó una prueba de Tukey.

\section{RESULTADOS Y DISCUSIÓN}

Los parámetros productivos de conejos que consumieron infusión de epazote no fueron diferentes al tratamiento control, respecto al peso final, ganancia total de peso, ganancia diaria de peso global y conversión alimenticia (Cuadro 1); Musa et al., (2009) observaron que al adicionar enzimas y probióticos, una mejora en la ganancia de peso y la conversión alimenticia; por otra parte, Sharma et al., (2016), mencionaron que al incluir en la engorda de conejos probióticos se mejoró los parámetros productivos. 
Respecto al consumo de alimento, los resultados encontrados mostraron que dicho consumo fue diferente en cada semana para cada concentración, pero en la semana 4, al incluir $10 \mathrm{~g}$ de epazote como infusión el consumo fue mayor $(\mathrm{P}<0.05)$ (Cuadro 2); en cambio el consumo de infusión fue similar en la semana 3 y 4 para cada concentración, pero significativamente diferente a las semanas 1 y $2(P<0.05)$. Con respecto a la concentración de epazote en infusión, no se encontraron diferencias significativas entre cada tratamiento (Cuadro 3).

\begin{tabular}{lccc}
\hline & \multicolumn{3}{c}{ Epazote } \\
Parámetros productivos & 0 & $g \mathrm{~g}^{-1}$ & 10 \\
\hline Peso inicial $(\mathrm{Kg})$ & $0.66 \pm 0.06$ & $0.682 \pm 0.06$ & $0.659 \pm 0.06$ \\
Peso final $(\mathrm{Kg})$ & $1.94 \pm 0.07$ & $1.879 \pm 0.07$ & $1.904 \pm 0.07$ \\
Peso total ganado $(\mathrm{Kg})$ & $1.28 \pm 0.04$ & $1.198 \pm 0.04$ & $1.245 \pm 0.04$ \\
Ganancia diaria de peso & $46 \pm 0.001$ & $43 \pm 0.001$ & $0.044 \pm 0.001$ \\
(g) & & & \\
Conversión alimenticia & $2.66 \pm 0.08$ & $2.71 \pm 0.08$ & $2.84 \pm 0.08$ \\
\hline
\end{tabular}

Cuadro 1. Parámetros productivos de conejos en engorda que consumieron infusión de epazote.

\begin{tabular}{ccccc}
\hline $\begin{array}{c}\text { Concentración de } \\
\text { epazote en la } \\
\text { infusión }\left(\mathrm{g} \mathrm{L} \mathrm{d}^{-1}\right)\end{array}$ & SEMANA 1 & SEMANA 2 & SEMANA 3 $^{\mathrm{B}}$ & SEMANA 4A $^{\mathrm{C}}$ \\
\hline 0 & $101+0.3^{\mathrm{a}}$ & $112+0.3^{\mathrm{a}}$ & $130+0.3^{\mathrm{a}}$ & $140+0.3^{\mathrm{ab}}$ \\
5 & $100+0.3^{\mathrm{a}}$ & $107+0.3^{\mathrm{a}}$ & $122+0.3^{\mathrm{a}}$ & $136+0.3^{\mathrm{b}}$ \\
10 & $101+0.3^{\mathrm{a}}$ & $120+0.3^{\mathrm{a}}$ & $137+0.3^{\mathrm{a}}$ & $147+0.3^{\mathrm{a}}$ \\
\hline \multicolumn{4}{c}{$\mathrm{a}, \mathrm{b}$ ó A,B,C,D= Literales diferentes indican diferencia estadísticamente significativa $(\mathrm{P}<0.05)}$.
\end{tabular}

Cuadro 2. Consumo de alimento durante la engorda de conejos que consumieron infusión de epazote.

El rendimiento de la canal no fue diferente entre tratamientos al incluir infusión de epazote, obteniendo un promedio de 53.67\% \pm 2.04 , lo cual coincide con Acosta (2016) con el uso de bloques nutricionales en la engorda de conejos; lo cual difiere con Sharma et al., (2016), quienes indican que el porcentaje de rendimiento de la canal de conejos se ve afectada por el uso de probióticos.

\begin{tabular}{ccccc}
\hline $\begin{array}{c}\text { Concentración de } \\
\text { epazote en la } \\
\text { infusión }\left(\mathrm{g} \mathrm{L} \mathrm{d}^{-1}\right)\end{array}$ & SEMANA 1 & SEMANA 2 & SEMANA 3 ${ }^{\mathrm{AB}}$ & SEMANA 4A $^{\mathrm{C}}$ \\
\hline 0 & $146+0.1^{\mathrm{a}}$ & $184+0.1^{\mathrm{a}}$ & $214+0.3^{\mathrm{a}}$ & $234+0.3^{\mathrm{a}}$ \\
5 & $146+0.3^{\mathrm{a}}$ & $205+0.3^{\mathrm{a}}$ & $241+0.3^{\mathrm{a}}$ & $263+0.3^{\mathrm{a}}$ \\
10 & $150+0.3^{\mathrm{a}}$ & $200+0.3^{\mathrm{a}}$ & $230+0.3^{\mathrm{a}}$ & $252+0.3^{\mathrm{a}}$ \\
\hline \multicolumn{4}{c}{ a ó A,B,C,D= Literales diferentes indican diferencia estadísticamente significativa $(\mathrm{P}<0.05)}$. \\
\multicolumn{4}{c}{ Cuadro 3. Consumo de infusión de epazote durante la engorda de conejos. }
\end{tabular}




\section{CONCLUSIÓN}

La utilización de infusión de epazote en la engorda de conejo no afecta los parámetros productivos, ni el rendimiento de la canal, lo que sugiere una alternativa para suplementar la dieta de conejos, por sus beneficios medicinales; debido a que no hubo mortalidad en estos animales sin utilizar antibióticos.

\section{IMPLICACIONES}

Debido a que la inclusión de infusión de epazote en la engorda de los conejos no afectó los parámetros productivos y el rendimiento en canal, al ser una planta medicinal; sería importante evaluar su efecto sobre la calidad de la carne y los productos cárnicos derivados de la misma.

\section{LITERATURA CITADA}

ACOSTA Cl. 2016. Evaluación de parámetros productivos y calidad de la canal en conejos de engorda alimentados con bloques nutricionales. Universidad Autónoma del Estado de Hidalgo. Tesis de Licenciatura. Tulancingo, Estado de Hidalgo. pp 58.

BLASCO A y Ouhayoun J. 1996. Harmonization of criteria and terminology in rabbit meat research. World Rabbit Science, 4:93-99.

FAO. 2015. Faostat. http//faostat3.fao.org/browse/Q/QL/S. Fecha de consulta: Octubre 2016.

MUSA HH, Wu SL, Zhu CH, Seri HI y Zhu GQ. 2009. The Potential Benefits of Probiotics in Animal Production and Health. Journal of Animal and Veterinary Advances, 8(2): 313321.

OLIVARES PR, Gómez CMÁ., Schewentesius RR y Carrera CB. 2009. Alternativas a la producción y mercadeo para la carne de conejo en Tlaxcala, México. Región y Sociedad. Septiembre-Diciembre, XXI (46), pp. 191-207.

PARA PA, Ganguly S, Wakchaure R, Sharma R, Mahajan T, y Praveen PK. 2015. Rabbit meat has the potential of being a possible alternative to other meats as a protein source: A brief review. Int J Phar Biomedi Res. 2: 17-19.

SHARMA KG, Vidyarthi VK, Archana K, Zuyie R. 2016. Probiotic supplementation in the diet of rabbits. A review. Livestock Research International. 4:1-10.

\section{AGRADECIMIENTOS}

El presente trabajo fue financiado por SEP-PROMEP con número de asignación DSA/103.5/16/10281. 